\section{International Scientific Journal Theoretical \& Applied Science}

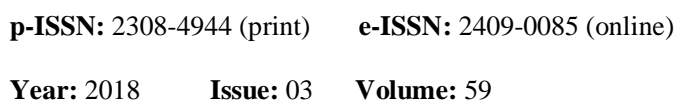

SECTION 20. Medicine
Ikilas Suyndikovich Moldaliyev Professor, Doctor of Medical Sciences International Kazakh-Turkish University by name Yassavi mikilas@mail.ru

Andrej Mechislavovich Grjibovski Phd, Dr.med Northern State Medical University, Arkhangelsk, Russia am.grzhibovskii@s-vfu.ru

Raimzhan Seitkhanuly Mamytkhan International Kazakh-Turkish University by name Yassavi raimjan_92@mail.ru

Gulbanu Zhandoskyzy Zhumadilla International Kazakh-Turkish University by name Yassavi banu_kds@mail.ru

Aygerim Amangeldievna Dosbergenova International Kazakh-Turkish University by name Yassavi aigerim dosbergenova1986@mail.ru

\title{
PROSPECTS FOR THE CREATION OF A POPULATION REGISTER OF INJURIES IN KENTAU REPUBLIC OF KAZAKHSTAN
}

\footnotetext{
Abstract: Analysis of the development prospects of the population register of injuries in Kentau, the development of a regional injury preventionprogram and accreditation in Kentau as the first safe community in Kazakhstan, the experience of which will be extended to the whole republic.

Key words: the population register of trauma,injury prevention, safe community.

Language: Russian

Citation: Moldaliyev IS, Grjibovski AM, Mamytkhan RS, Zhumadilla GZ, Dosbergenova AA (2018) PROSPECTS FOR THE CREATION OF A POPULATION REGISTER OF INJURIES IN KENTAU REPUBLIC OF KAZAKHSTAN. ISJ Theoretical \& Applied Science, 03 (59): 268-276.

Soi: http://s-o-i.org/1.1/TAS-03-59-46 Doi: crossef https://dx.doi.org/10.15863/TAS.2018.03.59.46

\section{УДК 616.8}

\section{ПЕРСПЕКТИВЫ СОЗДАНИЕ ПОПУЛЯЦИОННОГО РЕГИСТРА ТРАВМ В Г. КЕНТАУ РЕСПУБЛИКИ КАЗАХСТАН}

Аннотация: Анализ перспективы разработки популяционного регистра травм в 2. Кентау, разработки региональный программы профилактики травматизма и аккредитации г. Кентау в качестве первого в Казахстане безопасного сообщества, опыт которого будет распространен на всю Республику.

Ключевые слова: популячинный регистр травмы, профилактика травматизма, безопасное сообщества.
}

\section{Introduction}

Травмы составляют одну из ведущих проблем здравоохранения, занимая третье место среди причин смертности в Европейском регионе ВО3 после болезней органов кровообращения и злокачественных новообразований. Во всем мире более пяти миллионов человек умирает ежегодно вследствие травм [10]. По данным Росстата в
Российской Федерации травмы также занимают третье место в структуре причин смертности среди населения [2], однако уровень смертности, связанный с травмами в России более чем в два раза выше по сравнению со странами Евросоюза.

В Республике Казахстан травматизм одно из важных медико-социальных проблем. По общей заболеваемости травматизм после болезни 
сердечно-сосудистых систем занимает второе место. В Республике Казахстан каждый год на 100 тыс населения регистрируется 4 тыс травма, соответственно 600 тыс население получает травмы в год. Из них составляют 70\% взрослые, молодежы - 9\%, дети - $21 \%$.

Сегоднящей день в Республике Казахстан по показателям смертности травма после сердечнососудистых заболеваний занимает второе место.

Если анализировать показатель травматизма по городу Кентау Южно-Казахстанской области: если травму 2013 году получил 979 человек, то 2014 год - 1014, 2015 год - 1340, а 2016 году уже пострадал 2023 человек. По этим данным мы увидим, что показатель травматизма по городу Кентаупоследняя 4 года выросла боле 2 раза.

Травматизм оказывает существенное влияние на здоровье населения из-за тяжести социальных последствий, приводя к инвалидности, временной утрате трудоспособности, различным медицинским расстройствам и длительным психологическим эффектам.

Международный опыт показывает, что важным условием для разработки эффективной программы профилактики травм является наличие данных хорошего качества о травмах и обстоятельствах их получения [24]. В Казахстане данные обо всех случаях травм регистрируются лечебно-профилактическими учреждениями в соответствии с МКБ-10. Стандартно регистрируемые данные содержат основную медицинскую информацию: вид травмы, место и время её получения, характер и объём полученных телесных повреждений, вид деятельности, в ходе которого получена травма, предмет или вещество, контакт с которым привёл к её появлению, а также социальнодемографические данные травмированного (в основном, пол, и возраст). Эти данные позволяют учитывать и классифицировать травмы, определять их инцидентность, выделять группы высокого риска по полу и возрасту. Однако эти стандартно регистрируемые данные не представляют собой достаточной доказательной базы для планирования эффективных профилактических программ. Они содержат слишком мало информации о том, что именно являлось причиной конкретной травмы, в каких обстоятельствах она произошла, какие сопутствующие факторы обусловили собой её получение. Иными словами, стандартно регистрируемые данные дают недостаточно информации о факторах, устранение или изменение действия которых могло бы предотвратить возникновение новых случаев травм.

\section{Materials and Methods}

За рубежом начиная с 70-х годов XX века стали развиваться регистры травм, которые включают данные о пациентах, обратившихся за медицинской помощью по поводу травмы. Впервые компьютеризованная база данных о травмах была создана в США в 1969 году в госпитале округа Cook в Чикаго. В 1971 году в штате Иллинойс в сборе данных о травмах участвовали уже 50 больниц. В настоящее время в США регистры травм функционируют в 37 штатах. Вся информация, поступающая из регистров, консолидируется в Национальном банке данных о травмах (National TraumaDataBank, NTDB). Национальные популяционные регистры травм существуют также в Канаде [9], Австралии и Новой Зеландии [7]. В большинстве стран Западной Европы имеются действующие регистры травм (таблица). В 1990 году в регистр травм в Великобритании была введена информация о первом пациенте, к 2010 году база данных регистра включала 27000 пациентов, а информация поступала от 157 больниц. В Германии первый пациент с травмой был зарегистрирован в базе данных в 1993 году, а в 2012 году регистр насчитывал уже 28805 пациентов, информация о которых поступала от 573 больниц. Следует отметить, что в развивающихся странах существует много барьеров на пути к созданию регистров травм [15], тем не менее, в некоторых странах созданы и успешно функционируют региональные регистры травм [11, 12, 13, 16, 17, 18].

В создании регистра травм ключевую роль играют следующие элементы: 1) собираемые переменные, 2) критерии включения и исключения, 3) программное обеспечение для ведения регистра, 4) обученный персонал, 5) проверка качества данных [27]. Не существует общих или всеобъемлющих рекомендаций по развитию каждого элемента регистра травм. Каждая организация определяет свой регистр травм, основанный на ее потребностях, государственных или городских запросах. Часто в регистр травм включаются дополнительные переменные, зависящие от цели его создания.

Регистры травм дают возможность пользователям: 1) оценивать и улучшать лечение; 2) выявлять возможности для профилактических мероприятий; 3) документально подтверждать медицинские, экономические и социальные эффекты травмы; 4) разрабатывать и проверять исследовательские гипотезы [14, 27]. Регистры травм, созданные для клинических целей, содержат больше клинической информации, которая отсутствует в госпитальной административной базе данных, и поэтому чаще используются для оценки эффективности новых видов клинических вмешательств. Регистры 


\begin{tabular}{|c|c|c|c|c|c|c|}
\hline Impact Factor: & $\begin{array}{l}\text { ISRA (India) } \\
\text { ISI (Dubai, UAE } \\
\text { GIF (Australia) } \\
\text { JIF }\end{array}$ & $\begin{array}{r}=1.344 \\
=0.829 \\
=0.564 \\
=1.500\end{array}$ & $\begin{array}{l}\text { SIS (USA) } \\
\text { PИНЦ (Russia) } \\
\text { ESJI (KZ) } \\
\text { SJIF (Morocco) }\end{array}$ & $\begin{array}{l}=0.912 \\
=0.207 \\
=4.102 \\
=\mathbf{2 . 0 3 1}\end{array}$ & $\begin{array}{l}\text { ICV (Poland) } \\
\text { PIF (India) } \\
\text { IBI (India) }\end{array}$ & $\begin{array}{l}=6.630 \\
=1.940 \\
=4.260\end{array}$ \\
\hline
\end{tabular}

травм, созданные для профилактических вмешательств, включают информацию о механизме и обстоятельствах травмы. Эта информация может быть использована на местном, региональном или государственном уровнях для разработки вмешательств, направленных на снижение риска травм, например, изменение покрытия детской или спортивной площадки, использование шлема при езде на мотоцикле, ограничение допустимого уровня алкоголя в крови для водителей транспортных средств [5, 8, 19]. Данные о травмах, собранные за временной промежуток, могут быть использованы для оценки эффективности внедренных профилактических мероприятий, изменения уровня травматизма и смертности от него. Оценка эффективности профилактических мероприятий по данным регистра травм может быть осуществима с помощью экспериментального исследования на популяционном уровне, которое имеет самую высокую степень доказательной способности при надлежащем его проведении [3, 4].

Таблица1

Европейские регистры травм [6]

\begin{tabular}{|c|c|c|c|c|}
\hline Страна & Название регистра травм & $\begin{array}{c}\text { Количество } \\
\text { больниц, } \\
\text { предостав- } \\
\text { ляющих данные }\end{array}$ & $\begin{array}{c}\text { Количество } \\
\text { пациентов }\end{array}$ & Период* \\
\hline Германия & $\begin{array}{l}\text { TR-DGU Немецкое } \\
\text { травматологическое } \\
\text { общество, Кельн }\end{array}$ & 573 & 122742 & $1993-2012$ \\
\hline Великобритания & $\begin{array}{l}\text { Аудит травм и } \\
\text { исследовательская сеть } \\
\text { (TARN) }\end{array}$ & 175 & 27000 & $1990-2010$ \\
\hline Нидерланды & Голландский аудит травм & 80 & 43726 & $2007-2010$ \\
\hline Италия & $\begin{array}{l}\text { Итальянский } \\
\text { национальный регистр } \\
\text { основных травм (RITG) }\end{array}$ & 26 & 6583 & $2007-2013$ \\
\hline Дания & $\begin{array}{l}\text { TARN, Копенгаген и } \\
\text { регистр травм Южной } \\
\text { Дании, Оденсе }\end{array}$ & 4 & 1678 & $1996-2010$ \\
\hline Швейцария & TRAC - CHUV, Лозанна & 2 & 315 & $2008-2010$ \\
\hline Финляндия & $\begin{array}{l}\text { Тӧӧlö госпитальный } \\
\text { регистр травм, Хельсинки }\end{array}$ & 1 & 400 & $2006-2010$ \\
\hline Норвегия & $\begin{array}{l}\text { Регистр травм } \\
\text { университетской больницы } \\
\text { Ullevål, Осло }\end{array}$ & 1 & 1415 & $2000-2010$ \\
\hline Португалия & REGTRAUMA, Порто & 1 & 152 & $2001-2010$ \\
\hline Испания & $\begin{array}{l}\text { Регистр политравмы, } \\
\text { Каталония }\end{array}$ & - & 400 & $2001-2010$ \\
\hline
\end{tabular}

* за который зарегистрировано указанное количество пациентов.

Одним из положительных примеров ведения регистра травм является опыт г. Харстад в Норвегии, который показал, что создание городского регистра травм, собирающего в себе не только стандартную медицинскую информацию, но и подробную информацию о причинах и обстоятельствах всех травм, регистрируемых на территории города, позволяет 
разработать на его основе эффективные локальные профилактические программы [20-23, 25, 26]. Регистр травм в г. Харстад начал функционировать в 1985 году и к настоящему времени база данных содержит информацию более чем о 70000 случаев травм. В ходе долгосрочной работы в г. Харстад было достигнуто более чем двукратное сокращение общих показателей травматизма, а также сведение к нулю частоты отдельных видов травм. Использование данных регистра травм позволило разработать и внедрить популяционную программу профилактики, направленную на снижение дорожно-транспортного травматизма, что привело к снижению частоты травм среди детей на 59\%, а среди совокупного населения на $37 \%$ [24].

Базируясь на подходе «использование лучшего опыта» в 2014 году стартовал российско-норвежский проект «Популяционноориентированная программа профилактики и мониторинг травматизма в Шенкурске». Цель проекта заключалась в создании в г. Шенкурске Архангельской области муниципального (городского) регистра травм и разработке городской популяционно-ориентированной программы профилактики травм, с последующим присвоением г. Шенкурску статуса первого в России Безопасного Сообщества (SafeCommunity) и присоединением его к Международной Сети Безопасных Сообществ (International NetworkofSafeCommunities), ассоциированной с ВО3. Основная идея проекта состояла в том, что успешный опыт г. Харстад в области межведомственной профилактики травматизма на основе данных регистра травм, можно использовать и в Шенкурске с учетом местных условий и некоторой адаптации к ним. В 2017 году Шенкурск успешно прошел аккредитацию и стал первым на территории Таможенного Союза международно признанным безопасным сообществом. В ходе предполагаемого проекта опыт г. Харстад (Норвегия) и г. Шенкурск (Россия) будет использоваться для разработки популяционного регистра травм в г. Кентау, разработки региональный программы профилактики травматизма и аккредитации г. Кентау в качестве первого в Казахстане безопасного сообщества, опыт которого будет распространен на всю Республику.

Реализация проекта будет осуществляться в сотрудничестве МКТУ и Норвежского института общественного здравоохранения (Осло, Норвегия), Северного государственного медицинского университета (г. Архангельск), Норвежского центра содействия безопасности (NorsafetyAs, г. Харстад) и Шенкурского муниципального регистра травм (Россия).что Норвежский институт общественного здравоохранения несколько десятилетий назад уже поддерживал аналогичные проекты в г. Харстад и других городах Норвегии, а СГМУ патронировал создание регистра и организацию безопасного сообщества в г. Шенкурск, подтверждая тем самым достаточную компетентность и многолетний опыт участвующих партнеров в подобных проектах.

Работа по созданию регистра травм в г. Кентау, аналогичного городскому регистру травм в г. Харстад (Норвегия) и г. Шенкурск, будет включать в себя перевод с норвежского языка и русского на казахский формы по регистрации травмы (лист учета травм) и Руководства по ведению регистра травм с учетом местной и национальной специфики. В местный вариант листа учета травм будут добавлены вопросы, наиболее актуальные для Казахстана. Переведенное Руководство по ведению регистра травм будет содержать все необходимые определения, классификации и кодировочные листы, которые используются при формировании международных регистров травм. Ключевые классификации соответствуют «Североевропейской классификации для регистрации несчастных случаев», изданной Комитетом медицинской статистики Северной Европы (NOMESKO) в 1990 г. $\quad$ с целью формирования сопоставимых статистических данных в Европейских странах.

На следующем этапе проекта будет производиться формирование и ведение электронной базы регистра травм. Ответственные будут специально обучены принципам ввода данных, кодировки, сводки и обработки информации зарубежным специалистом. Предусматривается возможность стажировки на месте в одном из зарубежных регистров травм.

Для обеспечения высокого качества регистра будут проведены регулярные исследования валидности данных и логистики сбора и регистрации.

Пациенты подлежат регистрации, даже если им была оказана только элементарная или первая помощь, ранее оказана неотложная помощь или было проведено заведомо временное лечение в другом медицинском учреждении. Пациенты, обращающиеся повторно для контроля за ходом лечения, снятия гипса и т.п. после получения первичной помощи, не подлежат регистрации. Ранние осложнения травм, осложнения, полученные в ходе хирургических и терапевтических вмешательств, поздние последствия травм не подлежат регистрации (МКБ-10:Т79-98).

Каждый пациент, обратившийся за медицинской помощью в u- Rtynfe, по поводу травмы (включая, отравления) заполняет в день обращения или спустя несколько дней после 


\begin{tabular}{l|lr|ll|ll} 
& ISRA (India) & $=\mathbf{1 . 3 4 4}$ & SIS (USA) & $=\mathbf{0 . 9 1 2}$ & ICV (Poland) & $=\mathbf{6 . 6 3 0}$ \\
Impact Factor: & ISI (Dubai, UAE) $=\mathbf{0 . 8 2 9}$ & PUHЦ (Russia) $=\mathbf{0 . 2 0 7}$ & PIF (India) & $=\mathbf{1 . 9 4 0}$ \\
& GIF (Australia) & $\mathbf{0 . 5 6 4}$ & ESJI (KZ) & $=4.102$ & IBI (India) & $=\mathbf{4 . 2 6 0}$ \\
& JIF & $=\mathbf{1 . 5 0 0}$ & SJIF (Morocco) & $=\mathbf{2 . 0 3 1}$ & & \\
\hline
\end{tabular}

поступления на стационарное лечение лист учета травм самостоятельно, при помощи родственников или медицинской сестры. В случае, если пациент не может заполнить лист учета травм в связи с тяжелой степенью травмы или отказывается заполнять его, то данные о травмированном заносятся в лист ретроспективно регистратором травм по данным медицинской документации (журнал скорой помощи, карта амбулаторного пациента, история болезни) и информации, полученной от медицинского персонала, оказывавшего пациенту медицинскую помощь.

Информация о диагнозе (по МКБ-10) и степени тяжести травмы (по AIS) заполняется врачом. Данные листов учета травм ежедневно переносят в регистр медицинские сестры (регистраторы травм).

Первичное обращение пациента по поводу травмы в ЛПУ

(код по МКБ-10:S00 - Т78)

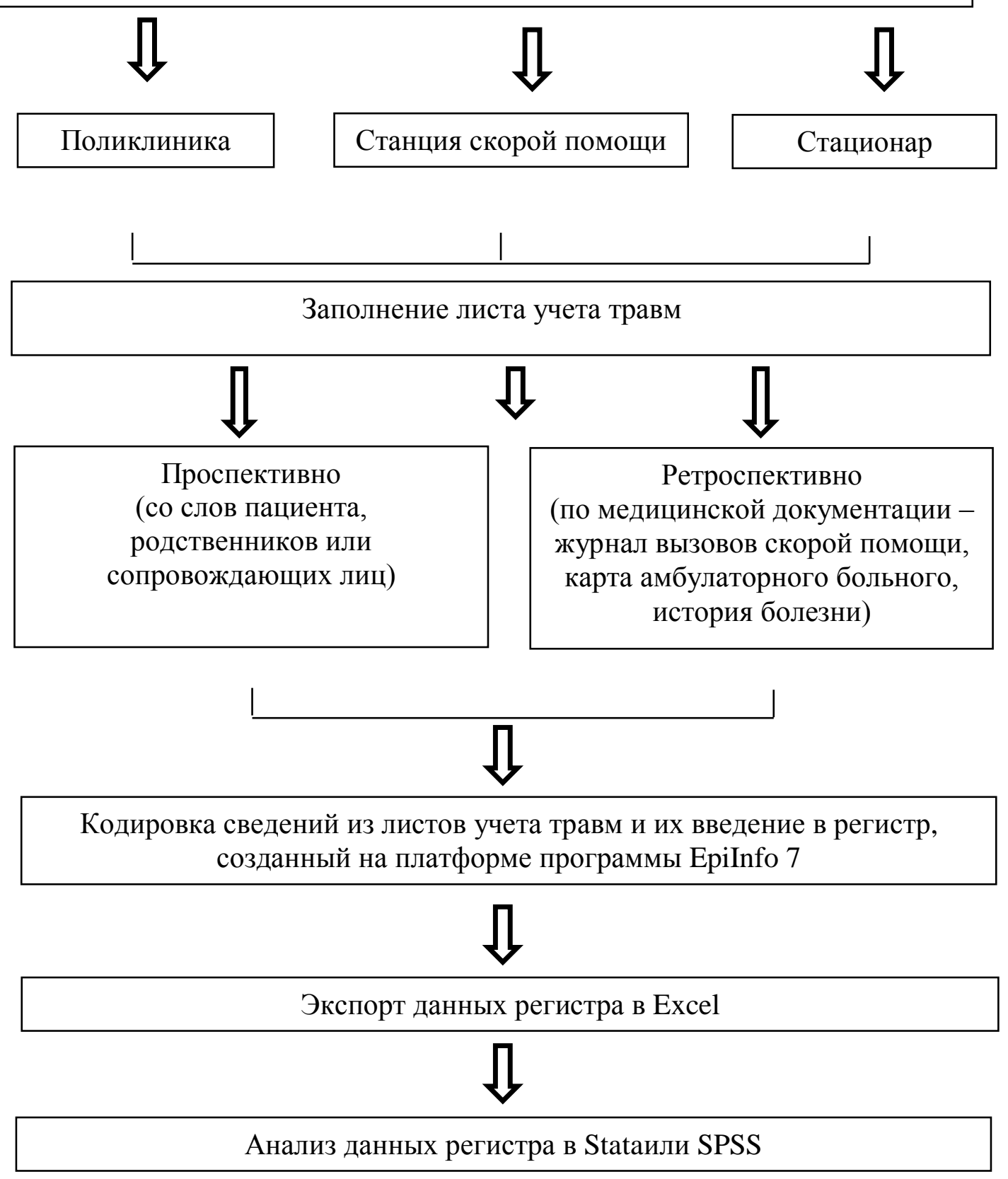

Рис. 1. Алгоритм работы Кентауского городского регистра травм 
Для получения информированного согласия пациентов на внесение в Регистр данных о полученных травмах на условиях сохранения их анонимности, с последующим использованием в целях профилактики травм и в научных целях, в стандартные формы информированного добровольного согласия на медицинское вмешательство был внесен соответствующий пункт.

Лист учета травм содержит разделы по сбору информации о типе, месте, предшествующих травме обстоятельствах, механизме травмы, потреблении алкоголя за 24 часа до травмы, социально-демографическую характеристику о травмированном. Фамилия, имя и отчество пациента, равно как и другие персональные данные, указываемые в листе учета травм (адрес, место работы/учебы, адрес проживания, номер телефона) не вносятся в регистр в целях обеспечении конфиденциальности персональной информации.
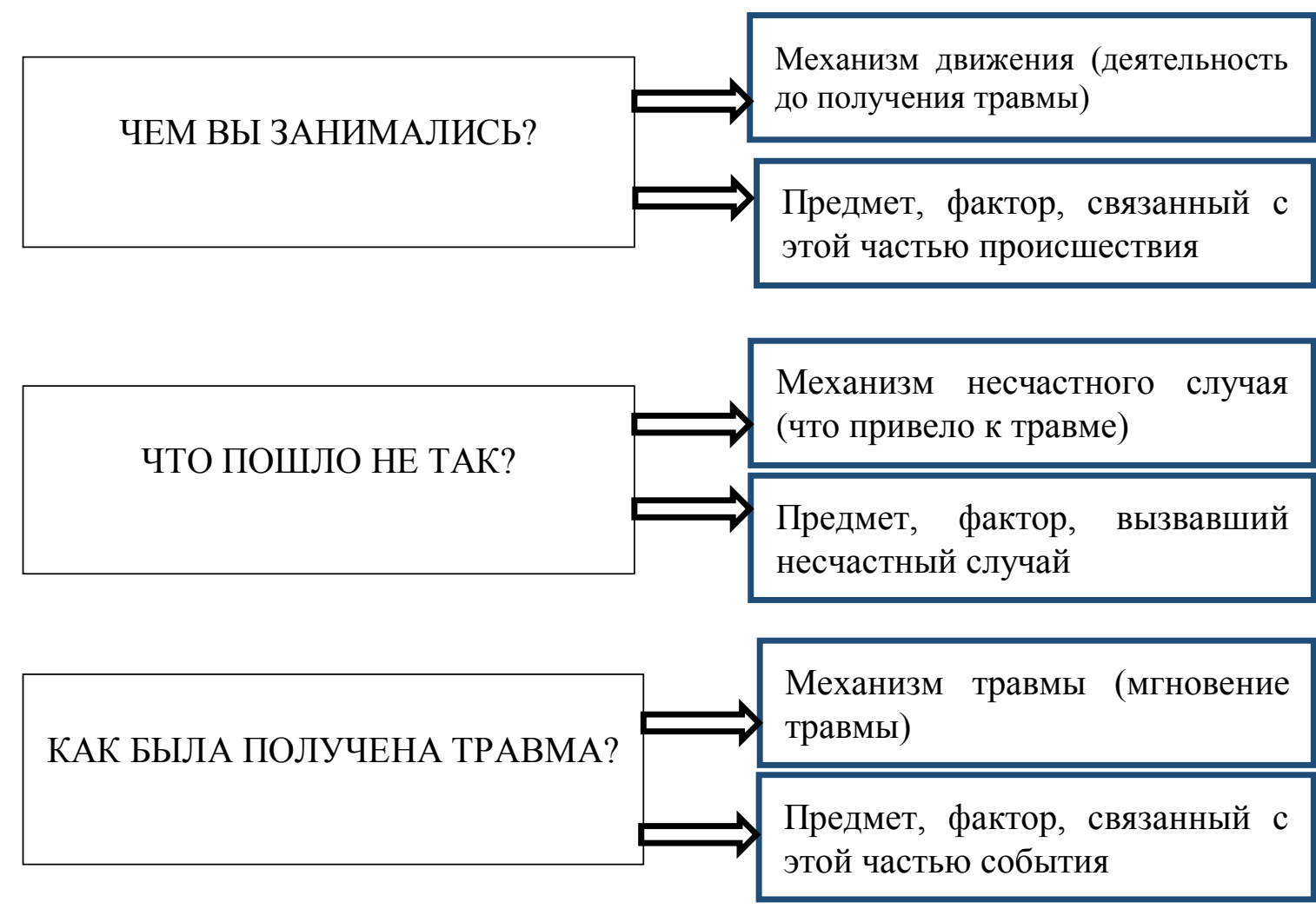

Рис. 2. Описание ситуации при возникновении травмы

В то же время, наличие персональных и контактных данных на листах учета травм, хранимых в качестве персональной медицинской документации в больнице, позволяет медицинскому персоналу связываться с пациентом для уточнения данных о полученной травме, если они не в полной мере указаны на листе учета травм. Особенность листов регистрации травм в том, что собираемая информация о характере полученных повреждений не ограничивается диагнозом и соответствующим кодом МКБ-10, а одновременно собираются подробные данные о том, чем занимался травмированный до получения травмы, как и в каких обстоятельствах произошел несчастный случай (что пошло не так, что этому способствовало), а также, как непосредственно была получена травма. Для установления последовательности событий при возникновении несчастного случая в листе учета травм есть поле, куда вносится описание ситуации, в результате которой возникла травма. Последовательность действий, которая привела к травме, представляется как обычный свободный текст, состоящий из трех взаимосвязанных вопросов (рис. 2), касающихся трех этапов: действий до получения травмы, механизма возникновения несчастного случая и непосредственно механизма получения травмы. Сформулированные вопросы имеют пояснения в 
скобках, указывающие на необходимость упоминания участвующих объектов, предметов, внешних факторов и иных важных обстоятельств на каждом из трех этапов.Именно эта информация важна, чтобы понять, что можно было сделать, чтобы предотвратить произошедшее, каким образом можно было деактивировать механизмы развития несчастного случая и получения травмы. Полнота и качество этой информации являются необходимыми условиями для разработки действительно эффективных программ профилактики местного уровня.

Программной основой системы регистрации травм является Epilnfo 7. Сотрудники, ответственные за ввод в регистр сведений, занесенных в листы учета травм, пройдут инструктаж по использованию EpiInfo 7. Программа является англоязычной, но имеет интуитивно понятный интерфейс и не представляется сложной в использовании лицам, имеющим хотя бы небольшой опыт работы с компьютером и прошедшим инструктаж по использованию программы в рамках заполнения регистра травм.

При вводе в регистр сведений, занесенных в листы учета травм, многие поля в программе являются обязательными - программа не позволит завершить регистрацию случая, если они не заполнены. Остальные поля являются опционными - необходимость их заполнения определяется ответами, внесенными в обязательные поля. Сотрудники, осуществляющие ввод данных, должны внимательно отслеживать ситуации, требующие заполнения опционных полей, и заполнять их соответственно. Часть полей, например, содержащие даты, имеют диапазоны допустимых значений, что снижает вероятность ошибок ввода данных. Экспорт данных регистра в Excel производится 1-го числа каждого месяца. Сформированные таблицы Excel именуются соответствующей датой, сохраняться в архиве и передаются сотруднику, ответственному за проведение анализа данных.

Специально подготовленный персонал (регистраторы травм) следит за тем, чтобы все пациенты с травмами, обращающиеся в больницу (амбулаторное и стационарное отделения, отделение скорой медицинской помощи) были зарегистрированы; за тем, чтобы сведения о пациенте и описание происшествия, которое стало причиной травмы, были как можно лучше представлены; а также за тем, чтобы информация интерпретировалась, кодировалась и вносилась в компьютерную программу в соответствии с правилами, изложенными в Руководстве по ведению регистра травм.
Ответственные за организацию сбора сведений о травмах обеспечивают проведение организационно-методической работы с врачами и средним медицинским персоналом о порядке сбора сведений о травмах в целях формирования и ведения регистра. Их задачей также является разъяснение другим медицинским работникам, для каких целей создается регистр, и какая информация является наиболее важной. Это мотивирует врачей, фельдшеров и другой медицинский персонал на выполнение дополнительной работы по обеспечению заполнения листов учёта травм, контролю полноты и качества указанных данных. Ещё одной важной задачей персонала является контакт с окружением пациента и органами власти на месте в целях передачи местных данных, которые станут основой профилактической работы и мероприятий, направленных на сокращение числа травм и несчастных случаев.

В помещениях ЛПУ, куда могут обратиться пациенты по поводу травм, будут размещены наглядные информационные стенды для населения о регистре травм, его целях и решаемых задачах, что позволяет повысить мотивацию пациентов по заполнению листов учета травм. Кроме того, мы будем через прессу регулярно публиковать анализ обобщенных данных регистра по актуальной тематике для определенного сезона.

\section{Conclusion}

Созданный в Кентау регистр травм будет научно-практической базой для решения вопросов, связанных с профилактикой травматизма. Ведение регистра позволяет наблюдать, предсказывать и минимизировать ущерб, нанесенный травмами, а также повышает наши знания относительно того, какие факторы вносят вклад в обстоятельства, вызывающие травмы. Выявление устранимых и модифицируемых факторов, являющихся частью механизма несчастных случаев и травм, позволит разработать целенаправленные меры профилактики по снижению частоты травм среди населения г. Кентау и ЮКО.

Для реализации задач проекта по популяционно-ориентированной программе профилактики и мониторинга травматизма будет создана межведомственная группа. В рамках межведомственного сотрудничества будут созданы рабочие группы по безопасности общественных мест, детской безопасности, безопасности пожилых, дорожной безопасности, профилактике насилия, профилактике суицидов, мониторингу травм, поддержки бизнеса и информационной поддержке. Такое социальное партнерство, когда власть, бизнес и гражданское 
общество взаимодействуют для достижения общей цели, укрепляет профилактическую программу и будет способствовать снижению уровня травматизма.

Предлагаемая модель доказательной и популяционно-ориентированной профилактики травм, основанная на успешном норвежском и опыте, имеет большой потенциал в отечественном контексте и способна доказать, что уровнем травматизма можно управлять. Целостный подход, при котором меры по предупреждению травмоопасных ситуаций имеют не меньшее значение, чем процесс ликвидации происшествий, системность в сфере обучения разных групп населения по формированию безопасной модели поведения, расстановка приоритетов для концентрации усилий на наиболее проблемных задачах, все это должно позволит достичь поставленных целей и обеспечить городу Кентау статус первого в Казахстане «Безопасного сообщества».В случае успешности проекта, приобретенный опыт профилактики травм в Кентаубудет предложен для использования в качестве модели для тиражирования в других городах Казахстана.

\section{References:}

1. Buleshova A.M., Buleshov M.A., Kudryavtsev A.V., Kuandykova A.K., et al. (2016) Epidemiologiya travmatizma v g. Shymket Yuzhno-Kazakhstanskoy oblasti Respubliki Kazakhstan: obosnovanie neobkhodimosti sozdaniya munitsipal'nogo registra travm // Ekologiya cheloveka. 2016. № 6. p. 55 - 61 .

2. (2015) Demograficheskiy ezhegodnik Rossii. 2015: Stat. sb./ Rosstat. -263 p.

3. Kholmatova K.K., Khar'kova O.A., Grzhibovskiy A.M. (2016) Eksperimental'nye issledovaniya $\mathrm{v}$ meditsine $\mathrm{i}$ zdravookhranenii: planirovanie, obrabotka dannykh, interpretatsiya rezul'tatov // Ekologiya cheloveka. 2016. № 11. -p. 50 - 58.

4. Kholmatova K.K., Khar'kova O.A., Grzhibovskiy A.M. (2016) Klassifikatsiya nauchnykh issledovaniy $\mathrm{v}$ zdravookhranenii // Ekologiya cheloveka. 2016. № 1. -p. 57 - 64.

5. Bernardo L.M., Gardner M.J., Seibel K. (2001) Playground injuries in children: a review and Pennsylvania Trauma Center experience // J SocPediatrNurs. 2001. N 6. p. $11-20$.

6. Beruan M., Stoica B., Negoi I., Tanase I., Gaspar B. (2014) Trauma Registry - A necessity of modern clinical practice // Chirurgia. 2014. Vol. 109, N 2. -p. 157 - 160.

7. Cameron P.A., Gabbe B.J., McNeil J.J., Finch C.F., Smith K.L., Cooper D.J., Judson R., Kossmann T. (2005) The trauma registry as a statewide quality improvement tool // J Trauma. 2005. Vol. 59, N 6. -p. 1469 - 1476.

8. Croce M.A., Zarzaur B.L., Magnotti L.J., Fabian T.C. (2009) Impact of motorcycle helmets and state laws on society's burden: a national study // Ann Surg. 2009. Vol. 250, N 3. p. $390-394$.

9. Datta I., Findlay Ch., Kortbeek J.B., Hameed S.M. (2007) Evaluation of a regional trauma registry // J Can Chir. 2007. Vol. 50, N 3. p. $210-213$.

10. (2014) Injuries and violence. The facts. WHO. 2014. 20 p.

11. Kobusingye O.C., Lett R.R. (2000) Hospitalbased trauma registries in Uganda // J Trauma. 2000. Vol. 48, N 3. p. 498 - 502.

12. Lakshmi P.V., Tripathy J.P, Tripathy N., Singh S., Bhatia D., Jagnoor J., Kumar R. (2016) A pilot study of a hospital-based injury surveillance system in a secondary level district hospital in India: lessons learnt and way ahead // InjEpidemiol. 2016. Vol. 3, N 1. P. $24-34$.

13. Mehmood A., Razzak J.A., Kabir S., MacKenzie E.J., Hyder A.A. (2013) Development and pilot implementation of a locally developed Trauma Registry: lessons learnt in a low-income country // BMC Emerg Med. 2013. Vol. 13. p. 4 - 11.

14. Moore L., Clark D.E. (2008) The value of trauma registries // Injury, Int. J. Care Injured. 2008. Vol. 39. p. $686-695$.

15. Nwomeh B.C., Lowell W., Kable R., Haley K., Ameh E.A. (2006) History and development of trauma registry: lessons from developed to developing countries // World J Emerg Surg. 2006. N 1. p. $32-40$.

16. O’Reilly G.M., Joshipura M., Cameron P.A., Gruen R. (2013) Trauma registries in developing countries: a review of the published 


\begin{tabular}{l|lr|ll|ll} 
& ISRA (India) & $=\mathbf{1 . 3 4 4}$ & SIS (USA) & $=\mathbf{0 . 9 1 2}$ & ICV (Poland) & $=\mathbf{6 . 6 3 0}$ \\
Impact Factor: & ISI (Dubai, UAE) $=\mathbf{0 . 8 2 9}$ & PUHЦ (Russia) $=\mathbf{0 . 2 0 7}$ & PIF (India) & $=\mathbf{1 . 9 4 0}$ \\
& GIF (Australia) & $\mathbf{0 . 5 6 4}$ & ESJI (KZ) & $=4.102$ & IBI (India) & $=\mathbf{4 . 2 6 0}$ \\
& JIF & $=\mathbf{1 . 5 0 0}$ & SJIF (Morocco) & $=\mathbf{2 . 0 3 1}$ & & \\
\hline
\end{tabular}

experience // Injury. 2013. Vol. 44, N 6. p. 713 - 721.

17. Parreira J.G., Campos T., Perungeiro J.A., Solda S.C., Assef J. C. et al. (2015) Implementation of the trauma registry as a tool for quality improvement in trauma care in a Brazilian hospital: the first 12 month // Rev Col Bras Cir. 2015. Vol. 42, N 4. p. 265 - 272.

18. Shaban S., Ashour M., Bashir M., El-Ashaal Y., Branicki F. et al. (2009) The long-term effects of early analysis of a trauma registry // World J Emerg Surg. 2009. N 4. p. $42-45$.

19. Stacey D.H., Doyle J.F., Gutowski K.A. (2008) Safety device use affects the incidence patterns of facial trauma in motor vehicle collisions: an analysis of the National Trauma Database from 2000 to 2004 // PlastReconstr Surg. 2008. Vol. 121. p. $2057-2064$.

20. Ytterstad B., Gordon S. S., Carolyn A. C. (1998) Harstad injury prevention study: prevention of burns in young children by community based intervention//Inj Prev. 1998. N 4. p. 176 - 180.

21. Ytterstad B., Wasmuth H.H. (1995) TheHarstad Injury Prevention Study: evaluation of hospitalbased injury recording and community-based intervention for traffic injury prevention // Accid Anal Prev.1995. Vol. 27, N 1. p. 111 123.

22. Ytterstad B. (1999) Harstad injury prevention study: the characteristics and distribution of fractures amongst elders--an eight year study //Int J Circumpolar Health. 1999. Vol. 8, N 2. p. $84-95$.

23. Ytterstad B. (1996) Harstad injury prevention study: the epidemiology of sports injuries. An 8 year study // Br J Sports Med. 1996. Vol. 30, N 1. p. 64 - 68 .

24. Ytterstad B. (2003) The Harstad Injury Prevention Study. A decade of communitybased traffic injury prevention with emphasis on children. Postal dissemination of local injury data can be effective // Int J Circumpolar Health. 2003. Vol. 62, N 1. p. 61 - 74.

25. Ytterstad B. (1996) The Harstad injury prevention study: community based prevention of fall-fractures in the elderly evaluated by means of a hospital based injury recording system in Norway // J Epidemiol Community Health. 1996. Vol. 50, N 5. p. $551-558$.

26. Ytterstad B. (1995) The Harstad injury prevention study: hospital-based injury recording used for outcome evaluation of community-based prevention of bicyclist and pedestrian injury // Scand J Prim Health Care. 1, 1995. Vol. 13, N 2. p.141 - 149.

27. Zehtabchi S., Nishijima D.K., McKay M.P., Mann N.C. (2011) Trauma registries: history, logistics, limitations, and contributions to emergency medicine research // AcadEmerg Med. 2011. Vol. 18, N 6. p. $637-643$. 